UVX 2012, 01016 (2013)

DOI: $10.1051 /$ uvx/201301016

(C) Owned by the authors, published by EDP Sciences, 2013

\title{
Étude du mécanisme d'échange et de la structure des matériaux hydroxydes doubles lamellaires (HDL) par diffraction et diffusion des rayons $X$
}

\author{
C. Taviot-Guého, F. Leroux, F. Goujon, P. Malfreyt et R. Mahiou
}

\author{
Clermont Université, Université Blaise Pascal, Institut de Chimie de Clermont-Ferrand, \\ BP. 10448, 63000 Clermont-Ferrand, France \\ CNRS, UMR 6296, ICCF, BP. 80026, 63171 Aubière, France
}

\begin{abstract}
Résumé. La maîtrise des performances des matériaux HDL (Hydroxydes Doubles lamellaires) nécessite une compréhension de plus en plus fine de leur structure ainsi que des relations entre structure, morphologie et propriétés d'emploi. Des mesures en diffraction et diffusion des rayons X (rayonnement synchrotron) haute résolution enregistrées à grand $\mathrm{Q}$ permettent d'aborder de manière plus précise la description structurale et la microstructure de ces matériaux mal cristallisés notamment via l'analyse de la fonction de distribution de paires (PDF). D'autre part, des mesures réalisées in situ en solution par diffraction des rayons en dispersion d'énergie (EDXRD) ont permis de révéler la formation de composés HDL biintercalés porteurs de nombreuses potentialités dans le domaine des matériaux multifonctionnels. Des mesures EDXRD ont également été appliquées à l'étude du mécanisme de formation de nanocomposites HDL-polymère qui représente un domaine d'application important des matériaux HDL.
\end{abstract}

\section{INTRODUCTION}

Avec de nombreuses applications possibles dans des domaines aussi variés que la catalyse, l'environnement, la santé et les polymères, les matériaux HDL (Hydroxydes Doubles Lamellaires), de formule générale $\left[\mathrm{M}^{2+}{ }_{1-x} \mathrm{M}^{3+}{ }_{x}(\mathrm{OH})_{2}\right]\left[\mathrm{X}^{\mathrm{m}-}{ }_{\mathrm{x} / \mathrm{m}}, \mathrm{nH}_{2} \mathrm{O}\right]$, font actuellement l'objet d'importantes recherches. En effet, de par leur richesse de composition tant au niveau des métaux $\left(\mathrm{M}^{2+}, \mathrm{M}^{3+}\right)$ constitutifs des feuillets hydroxydes que des espèces interlamellaires $\left(\mathrm{X}^{\mathrm{m}-}\right)$, également en raison de leur capacité d'échange anionique élevée, les HDL sont particulièrement adaptés à l'élaboration de matériaux multifonctionnels [1]. À cette malléabilité chimique et structurale, il faut aussi ajouter les nombreuses voies de synthèse et possibilités de mise en forme de ces matériaux qui agrandissent leur champ d'application.

La maîtrise des performances de ces matériaux nécessite une compréhension de plus en plus fine de leur structure ainsi que des relations entre la synthèse, la structure, la morphologie et les propriétés d'emploi. Deux études menées récemment dans l'équipe Matériaux Inorganiques de l'Institut de Chimie de Clermont-Ferrand (ICCF) ont permis d'enrichir le front des connaissances de la structure des phases HDL. Elles reposent sur l'utilisation du rayonnement synchrotron qui permet d'aborder de manière plus précise la description structurale et la microstructure de ces matériaux mal cristallisés ; un second aspect, associé à la puissance des flux, est le suivi in situ des processus de formation de ces matériaux.

This is an Open Access article distributed under the terms of the Creative Commons Attribution License 2.0, which permits unrestricted use, distribution, and reproduction in any medium, provided the original work is properly cited. 


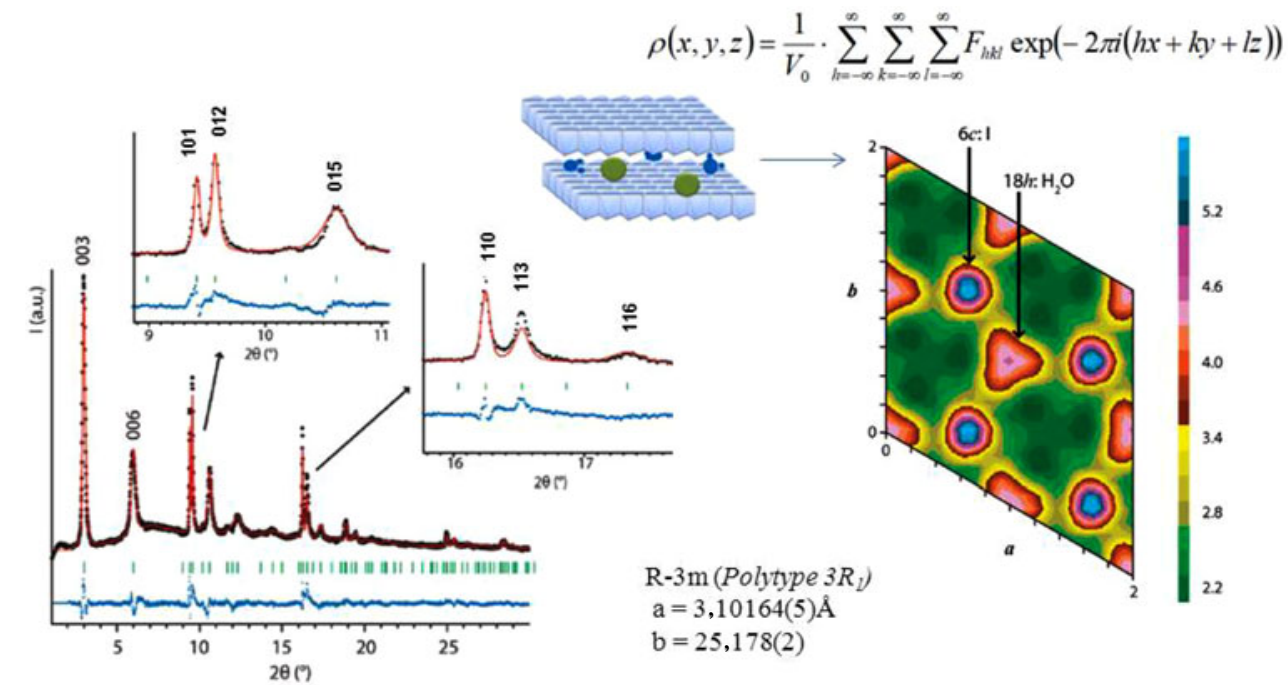

\begin{tabular}{|c|c|c|c|c|c|c|c|}
\hline Rietveld & atom & site & $\boldsymbol{x}$ & $y$ & $z$ & $B_{1 \infty}\left(A^{2}\right)$ & occupancy \\
\hline \multirow[t]{2}{*}{$R_{\text {op }}(\%)=7,6 / 18,1$} & $\mathrm{Zn}$ & $3 a$ & 0 & 0 & 0 & $0,64(3)$ & 0,0667 \\
\hline & Al & $3 a$ & 0 & 0 & 0 & $0,64(3)$ & 0,0167 \\
\hline \multirow{3}{*}{$\begin{array}{l}R_{\mathrm{Br} a g 8} / R_{\mathrm{f}}(\%)=6,4 / 5,5 \\
x^{2}=1,4\end{array}$} & $\mathrm{O}_{\mathrm{OH}}$ & $6 c$ & 0 & 0 & $0,3778(2)$ & $0,64(3)$ & 0,1667 \\
\hline & I & $\Leftrightarrow c$ & 0 & 0 & 0,16667 & 8,00 & 0,0167 \\
\hline & $\mathrm{O}_{w}$ & $18 \mathrm{~h}$ & $0,079(9)$ & $-0,079(9)$ & 0,5 & 8,00 & $0,0366(8)$ \\
\hline
\end{tabular}

Figure 1. Étude de la nanostructure du composé $\left[\mathrm{Zn}_{4} \mathrm{Al}(\mathrm{OH})_{10}\right]\left[\mathrm{I} \cdot 2,2 \mathrm{H}_{2} \mathrm{O}\right]$ : affinement Rietveld de données de diffraction X sur poudre $(\lambda=0,4368 \AA$ A ) enregistrées sur la ligne Cristal du synchrotron SOLEIL.

\section{2. ÉTUDE DE LA STRUCTURE DES COMPOSÉS HDL PAR DIFFRACTION ET DIFFUSION DES RAYONS X HAUTE RÉSOLUTION [2-5]}

Différents aspects de la structure des phases HDL ont pu être déterminés à partir de mesures en diffraction et diffusion des rayons $\mathrm{X}$ enregistrées à grand $\mathrm{Q}$ sur la ligne Cristal du synchrotron SOLEIL : la structure moyenne par la méthode de Rietveld, la microstructure via l'analyse du profil des raies de diffraction, le désordre structural et la présence de défauts via l'analyse de la fonction de distribution de paires (PDF). Les mesures discutées ci-après ont été réalisées sur le diffractomètre 2-cercles de la ligne Cristal en mode capillaire, équipé d'un multi-analyseur à 21 cristaux de $\mathrm{Si}(111)$ qui permet l'enregistrement simultané de 21 diagrammes de diffraction ; un faisceau monochromatique de longueur d'onde $\lambda=0,4368 \AA(28,2518 \mathrm{keV})$ a été utilisé donnant accès à un grand domaine de $\mathrm{Q}$ (vecteur de diffusion : $4 \sin \theta / \lambda): \mathrm{Q}_{\max }=25,4 \AA^{-1}$.

Ainsi, pour le composé $\left[\mathrm{Zn}_{4} \mathrm{Al}(\mathrm{OH})_{10}\right]\left[\mathrm{I} .2,2 \mathrm{H}_{2} \mathrm{O}\right]$ étudié dans le cadre du stockage de déchets nucléaires [? ], l'ajustement de ces données de diffraction avec l'algorithme de Rietveld (Programme FULLPROF [6]) a permis le calcul d'une carte de densité électronique détaillée pour l'espace interfeuillet à partir de laquelle la structure a pu être affinée (Figure 1). Le positionnement des anions $\mathrm{I}^{-}$sur le site cristallographique $6 \mathrm{c}$ (groupe d'espace $\mathrm{R}-3 \mathrm{~m}$ ), situé à mi-distance entre deux feuillets consécutifs et à l'aplomb des cations du feuillet, est en faveur d'interactions électrostatiques entre l'anion $\mathrm{I}^{-}$et le feuillet hydroxyde plutôt que de liaisons de type hydrogène, ce qui est en accord avec la polarisabilité élevée de cet anion.

Les phases HDL présentent des effets de distributions de taille de domaine de cohérence et de distorsion du réseau cristallin à l'origine d'élargissements importants des raies de diffraction. 

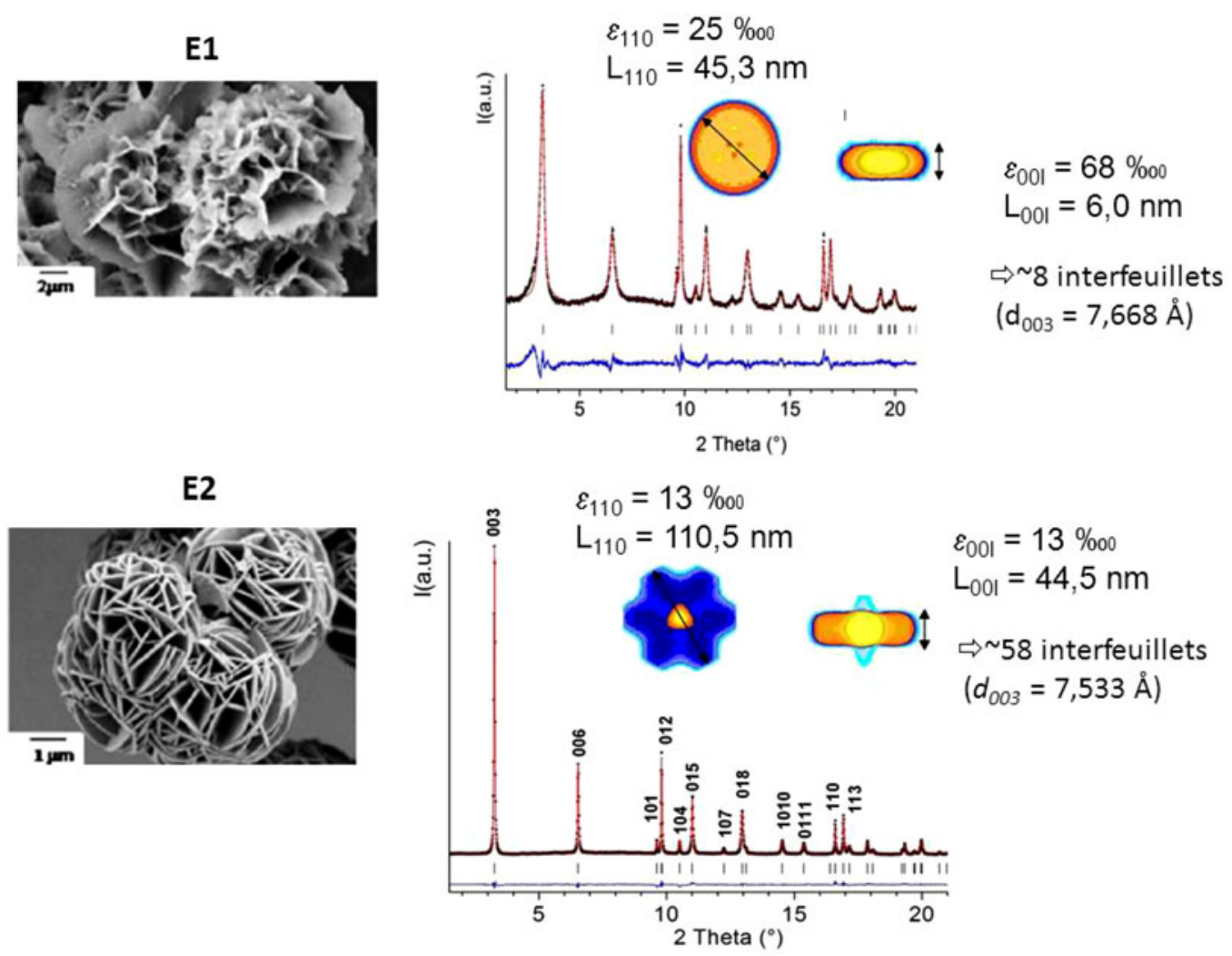

Figure 2. Étude de la microstructure des composés $\left[\mathrm{Ni}_{1,5} \mathrm{Al}_{0,5}(\mathrm{OH})_{6}\right]\left[1 / 4 \mathrm{CO}_{3}^{2-}, 2 \mathrm{H}_{2} \mathrm{O}\right]$ à partir de données de diffraction $\mathrm{X}$ sur poudre ( $\lambda=0,4368 \AA$, Cristal -synchrotron SOLEIL) ; ajustement du profil global avec la fonction Thompson Cox-Hastings pseudo-Voigt modifiée (TCH-Z).

La fonction de profil Thompson Cox-Hastings pseudo-Voigt modifiée (TCH-Z) [7] implémentée dans le programme d'affinement structural sur poudre FULLPROF [6] prend en compte ces effets et permet l'étude de la microstructure. Sur la Figure 2, sont représentés les domaines de cohérence construits à partir des paramètres affinés de la fonction TCH-Z pour deux composés HDL (notés E1 et E2) de même composition chimique $\left[\mathrm{Ni}_{1,5} \mathrm{Al}_{0,5}(\mathrm{OH})_{6}\right]\left[1 / 4 \mathrm{CO}_{3}^{2-}, 2 \mathrm{H}_{2} \mathrm{O}\right]$ obtenus par la méthode de synthèse « solvothermale » et présentant des morphologies très différentes [3,4]. La forme de ces domaines de cohérence rappelle la forme plaquettaire des cristaux avec notamment des épaisseurs tout à fait comparables à celles déterminées par analyse par microscopie électronique à transmission (MET). La taille des particules est donc probablement la seule à l'origine de l'élargissement des pics de diffraction $\mathrm{X}$ observé selon la direction de $00 l$ qui correspond à la direction d'empilement des feuillets ; par contre, les dimensions dans le plan sont largement inférieures à celles mesurées par MET indiquant une croissance latérale par coalescence non-cohérente des domaines adjacents. On note par ailleurs un taux de micro-contraintes relativement élevé pour le composé E1, selon la direction d'empilement des feuillets, qui est attribué à un phénomène d'interstratification $\left(\mathrm{CO}_{3}^{2-} / \mathrm{O}_{4}^{2-}\right)$.

Ces données synchrotron enregistrées à grand $\mathrm{Q}$ permettent également une étude de la fonction de distribution de paires (PDF Pair Distribution Function) [8]. La caractéristique principale de la PDF est de fournir une représentation de la structure d'un composé à travers la distribution des distances interatomiques et ceci dans une grande gamme d'échelles allant de quelques Å (ordre local) à plusieurs 


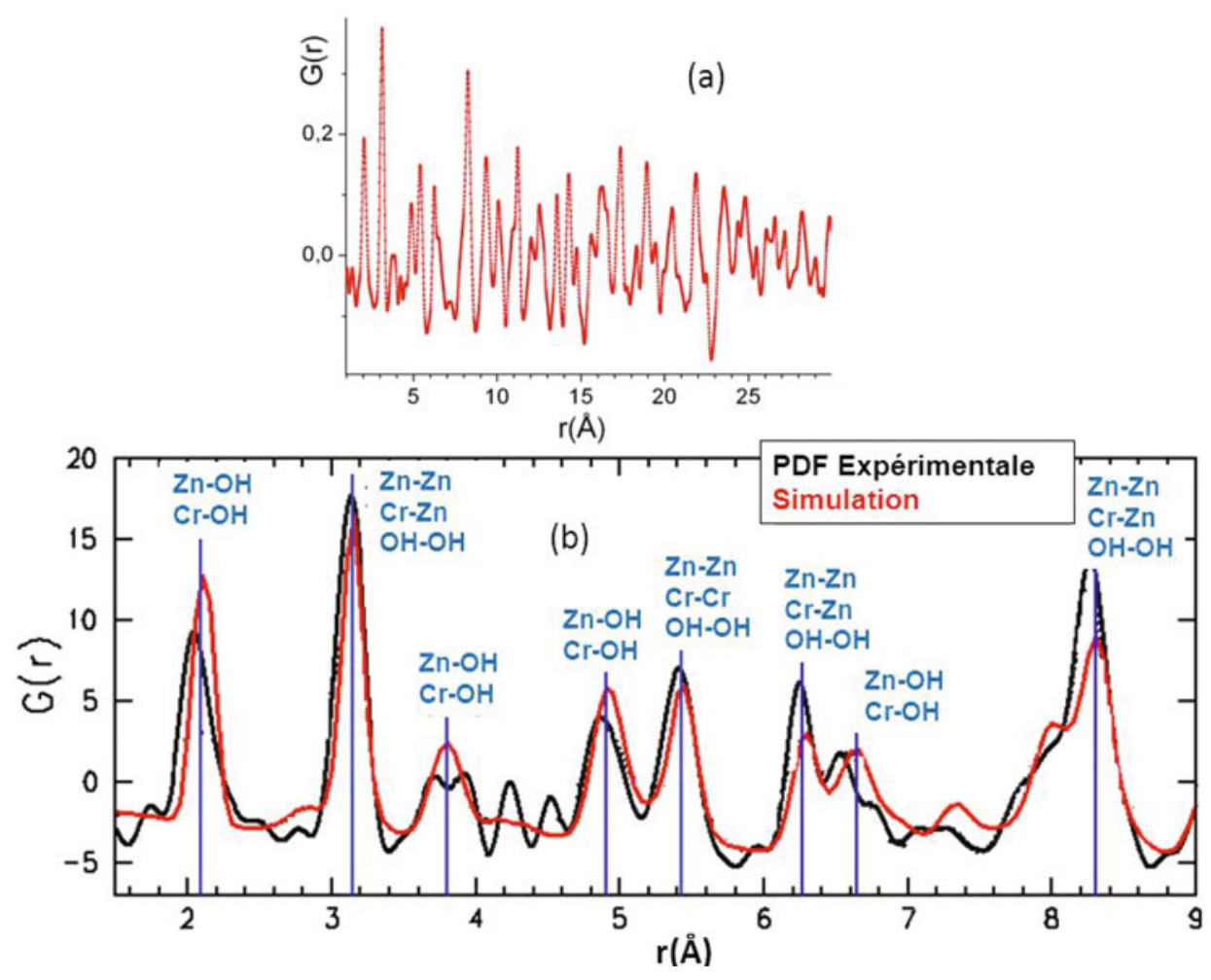

Figure 3. Analyse de la fonction de distribution de paires de $\left[\mathrm{Zn}_{2} \mathrm{Cr}(\mathrm{OH})_{6}\right]\left[\mathrm{Cl}, 2 \mathrm{H}_{2} \mathrm{O}\right]$ : (a) $\mathrm{PDF}$ expérimentale obtenue à partir de données de diffraction et de diffusion des rayons $\mathrm{X}$ enregistrées à grand $\mathrm{Q}\left(\mathrm{Q}_{\max }=25,4 \AA^{-1}\right.$, $\lambda=0,4368 \AA, 28,2518 \mathrm{keV}$, Cristal -synchrotron SOLEIL); (b) Détail des contributions obtenu par simulation de dynamique moléculaire.

dizaines de nm. La PDF peut être calculée à partir d'un modèle structural selon la formule suivante :

$$
G_{c}(r)=\frac{1}{r} \sum_{i} \sum_{j}\left[\frac{b_{i} b_{j}}{b^{2}} \delta\left(r-r_{i j}\right)\right]-4 \pi r \rho_{0}
$$

avec $r_{i j}$ distance inter atomique, $b_{i}$ : pouvoir diffusant de l'atome i, $\langle b>$ : facteur de diffusion moyen de l'échantillon et $\rho_{0}$ : densité numérique du composé.

Expérimentalement, la PDF s'obtient à partir d'un diffractogramme de poudre de l'échantillon étudié, en effectuant la transformée de Fourier de la fonction de structure de diffusion statique $S(Q)$ qui n'est autre que le diagramme de diffraction corrigé des contributions incohérentes et normalisé :

$$
G(r)=\frac{2}{z} \int_{0}^{Q}[S(Q)-1] \sin (Q r) d Q
$$

Dans le cas présent, le programme PDFGetX2 [9] a été utilisé pour le calcul de la PDF à partir de données de diffraction X sur poudre ainsi que le programme PDFFIT [10] pour les affinements et simulations. Prenant en compte à la fois les pics de Bragg et la diffusion diffuse entre les pics de Bragg, la PDF contient des informations sur le désordre structural et les défauts localisés ; elle est aussi appliquée à l'étude des arrangements atomiques dans les grains de taille nanométrique.

Pour l'interprétation de la fonction de distribution de paires du composé $\mathrm{HDL}\left[\mathrm{Zn}_{2} \mathrm{Cr}(\mathrm{OH})_{6}\right][\mathrm{Cl}$, $2 \mathrm{H}_{2} \mathrm{O}$ ], nous avons fait appel à la simulation de dynamique moléculaire en comparant la fonction de 


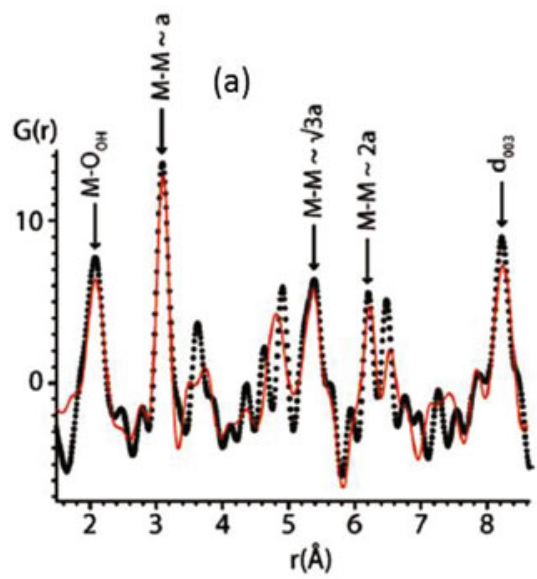

(b)

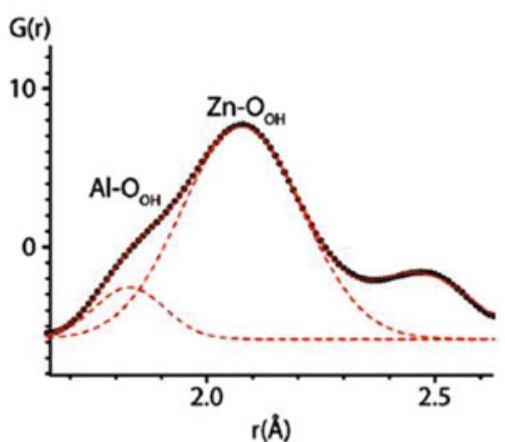

Figure 4. Fonction de distribution de paires du composé $\left[\mathrm{Zn}_{4} \mathrm{Al}(\mathrm{OH})_{10}\right]\left[\mathrm{I} \cdot 2,2 \mathrm{H}_{2} \mathrm{O}\right]$ (données expérimentales en noir et calculées en rouge) ; la PDF expérimentale a été obtenue à partir de données de diffraction et de diffusion des rayons $\mathrm{X}$ enregistrées à grand $\mathrm{Q}\left(\mathrm{Q}_{\max }=25,4 \AA^{-1}, \lambda=0,4368 \AA, 28,2518 \mathrm{keV}\right.$, Cristal -synchrotron SOLEIL).

distribution de paires obtenue expérimentalement à la PDF calculée à partir des configurations issues de dynamique moléculaire [5]. Cette approche combinée a permis l'identification de l'ensemble des distances interatomiques pour la PDF expérimentale (Figure 3); elle a également permis de révéler un ordre des cations $\mathrm{Zn}$ et $\mathrm{Cr}$ dans le feuillet hydroxyde ainsi que l'inter croissance de deux polytypes $2 \mathrm{H}_{1}$ et $3 \mathrm{R}_{1}$ pour l'échantillon analysé.

Pour le composé $\left[\mathrm{Zn}_{4} \mathrm{Al}(\mathrm{OH})_{10}\right]\left[1.2,2 \mathrm{H}_{2} \mathrm{O}\right]$ [? ], l'analyse de la fonction de distribution de paires indique un désordre total des cations $\mathrm{Zn}$ et $\mathrm{Al}$ dans le feuillet hydroxyde (Figure 4a). Pour ce composé, il a également été possible d'observer la contribution des 2 paires $\mathrm{Zn}-\mathrm{OH}$ et $\mathrm{Al}-\mathrm{OH}$, pourtant très proches d'un point de vue distance inter-atomique, 1,86 ̊̊ et 2,08 ̊̊ respectivement (Figure 4b).

Enfin, dans la cadre de l'étude de la relation structure-propriétés d'emploi des composés $\left[\mathrm{Ni}_{1,5} \mathrm{Al}_{0,5}(\mathrm{OH})_{6}\right]\left[1 / 4 \mathrm{CO}_{3}^{2-}, 2 \mathrm{H}_{2} \mathrm{O}\right]$, l'analyse de la fonction de distribution de paires nous permet d'affirmer que l'intercroissance des polytypes $2 \mathrm{H}_{1}$ et $3 \mathrm{R}_{1}$ pour ce système est un paramètre déterminant à l'origine de propriétés électrochimiques améliorées (Figure 5) [4].

\section{3. ÉTUDE DU MÉCANISME D'ÉCHANGE PAR DIFFRACTION DES RAYONS X EN DISPERSION D'ÉNERGIE [11-15]}

Afin de progresser dans la connaissance du mécanisme de l'échange anionique des phases HDL qui constitue une voie d'accès à une large gamme de matériaux hybrides HDL, nous avons réalisé une étude structurale et cinétique des réactions d'échange par diffraction des rayons $X$ en dispersion d'énergie (EDXRD Energy Dispersive X-ray Diffraction) [12]. Les mesures ont été réalisées sur le synchrotron SRS à Daresbury en Angleterre, sur la ligne 16,4. Les réactions d'échange ont lieu dans un tube de laboratoire $(\varnothing: 5 \mathrm{~cm})$ placé sur un agitateur magnétique et installé sur le trajet du faisceau blanc de rayons $\mathrm{X}\left(5-120 \mathrm{keV} ; 13 \mathrm{keV}=3,10^{10}\right.$ photons $\left./ \mathrm{sec}\right)$. $250 \mathrm{mg}$ d'HDL dispersés dans $10 \mathrm{~mL}$ d'eau sont initialement introduits dans la cellule ; l'ajout de la solution d'anion est réalisé à l'aide d'un pousse seringue. Le faisceau diffracté est analysé en énergie par un détecteur au germanium positionné à un angle $2 \Theta$ fixe de $1,625^{\circ}$. Une énergie de faisceau comprise entre 22 et $115 \mathrm{keV}$ a permis de collecter des spectres dans le domaine de distances inter-réticulaires $d$ 20-3,8 $\AA$ avec des temps d'acquisition de 10 à $60 \mathrm{sec}$. 


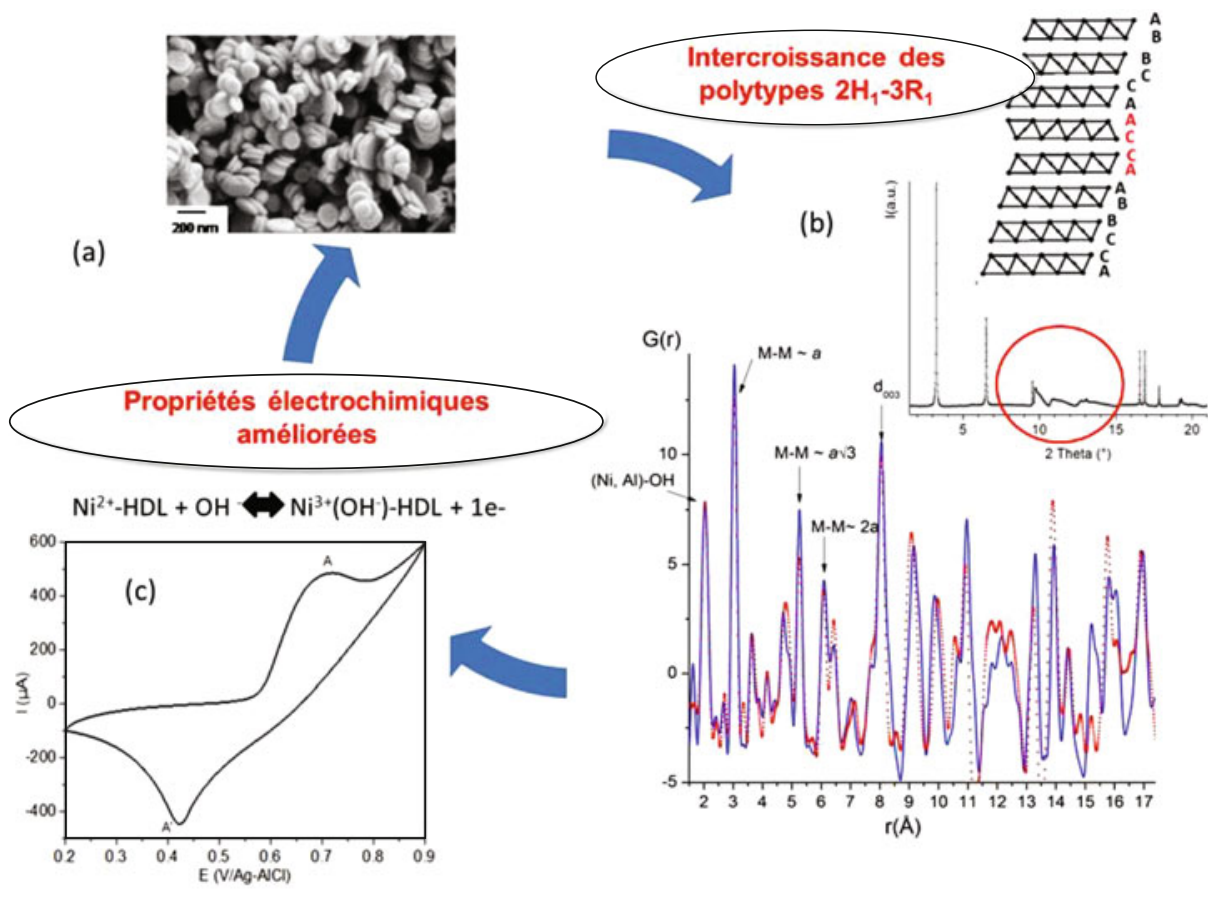

Figure 5. Étude de la relation synthèse-microstructure-propriété dans le cas du système $\left[\mathrm{Ni}_{1,5} \mathrm{Al}_{0,5}(\mathrm{OH})_{6}\right]$ $\left[1 / 4 \mathrm{CO}_{3}^{2-}, 2 \mathrm{H}_{2} \mathrm{O}\right]$ : (a) microscopie électronique MEB/MET, (b) données de diffraction $\mathrm{X}$ et fonction de distribution de paires enregistrées en capillaire avec $\lambda=0,4388 \AA\left(\mathrm{Q}_{\max }=25 \AA^{-1}\right)$ et (c) voltammétrie cyclique sur électrodes modifiées. La PDF expérimentale a été obtenue à partir de données de diffraction et de diffusion des rayons $\mathrm{X}$ enregistrées à grand $\mathrm{Q}\left(\mathrm{Q}_{\max }=25,4 \AA^{-1}, \lambda=0,4368 \AA, 28,2518 \mathrm{keV}\right.$, Cristal -synchrotron SOLEIL).

Le suivi in situ par EDXRD des réactions d'échange dans les phases $\mathrm{Zn}_{2} \mathrm{Al}-\mathrm{X}$ et $\mathrm{Zn}_{2} \mathrm{Cr}-\mathrm{X}$ en présence d'anions inorganiques $\mathrm{X}=\mathrm{Cl}^{-}, \mathrm{F}^{-}, \mathrm{CO}_{3}^{2-}, \mathrm{SO}_{4}^{2-}, \mathrm{Fe}(\mathrm{CN})_{6}^{3-}$ et organiques $\mathrm{X}=$ tartrate $\left({ }^{-} \mathrm{O}_{2} \mathrm{C}(\mathrm{CHOH})_{2} \mathrm{CO}_{2}^{-}\right)$, succinate $\left({ }^{-} \mathrm{O}_{2} \mathrm{C}\left(\mathrm{CH}_{2}\right)_{2} \mathrm{CO}_{2}^{-}\right)$, adipate $\left({ }^{-} \mathrm{O}_{2} \mathrm{C}\left(\mathrm{CH}_{2}\right)_{4} \mathrm{CO}_{2}^{-}\right)$, VBS (styrene-4sulfonate) a ainsi permis de révéler la présence de deux mécanismes d'échange : soit l'échange est direct avec la formation immédiate du composé totalement échangé, soit la réaction procède en deux temps avec la formation d'un composé intermédiaire obtenu par l'échange d'un interfeuillet sur deux. [14] Sur la Figure 5 sont reportées les mesures EDXRD pour la réaction d'échange des anions chlorure par les anions tartrate dans la phase $\mathrm{Zn}_{2} \mathrm{Cr}(\mathrm{OH})_{6} \mathrm{Cl} . n \mathrm{H}_{2} \mathrm{O}$. Dans le domaine d'énergie exploré, seules les réflexions $\mathbf{0 0 3}$ des phases $\mathrm{Zn}_{2} \mathrm{Cr}$-Cl et $\mathrm{Zn}_{2} \mathrm{Cr}$-Tart (produit final) et la réflexion 006 du composé intermédiaire bi-intercalé sont visibles. La solution d'anion tartrate $0,2 \mathrm{M}$ est additionnée à la vitesse de $0,059 \mathrm{~mL} / \mathrm{min}$ et le temps d'acquisition de chaque spectre est de $60 \mathrm{sec}$. Au fur et à mesure de l'ajout d'anions tartrate, la réflexion $\mathbf{0 0 3}$ de la phase initiale $\mathrm{Zn}_{2} \mathrm{Cr}-\mathrm{Cl}(7,8 \AA)$ disparaît lentement pour laisser place à la réflexion 006 du composé intermédiaire de stade $2 \mathrm{Zn}_{2} \mathrm{Cr}$-Cl/Tart $(10,1 \AA)$ qui disparaît à son tour progressivement au profit du composé final totalement échangé $\mathrm{Zn}_{2} \mathrm{Cr}$-Tartrate $\left(\begin{array}{llll}003 & 12,2 & \AA\end{array}\right)$. La position de la réflexion 006 du composé intermédiaire conduit à une distance interlamellaire pour celui-ci de $\boldsymbol{d}_{003}=2 * \boldsymbol{d}_{006}=20,2 \AA$ qui correspond à la somme des deux distances interlamellaires de la phase initiale $7,8 \AA$ et du produit final $12,2 \AA$.

La tendance générale est que l'échange d'anions inorganiques par d'autres anions inorganiques est direct alors que l'échange d'anions inorganiques par des anions organiques souvent procède en deux temps. Il semble donc raisonnable de penser que l'intercalation par stade dans le système HDL serait une particularité de l'échange inorganique-organique. Cette alternance de contenus interlamellaires de 


\section{UVX 2012}
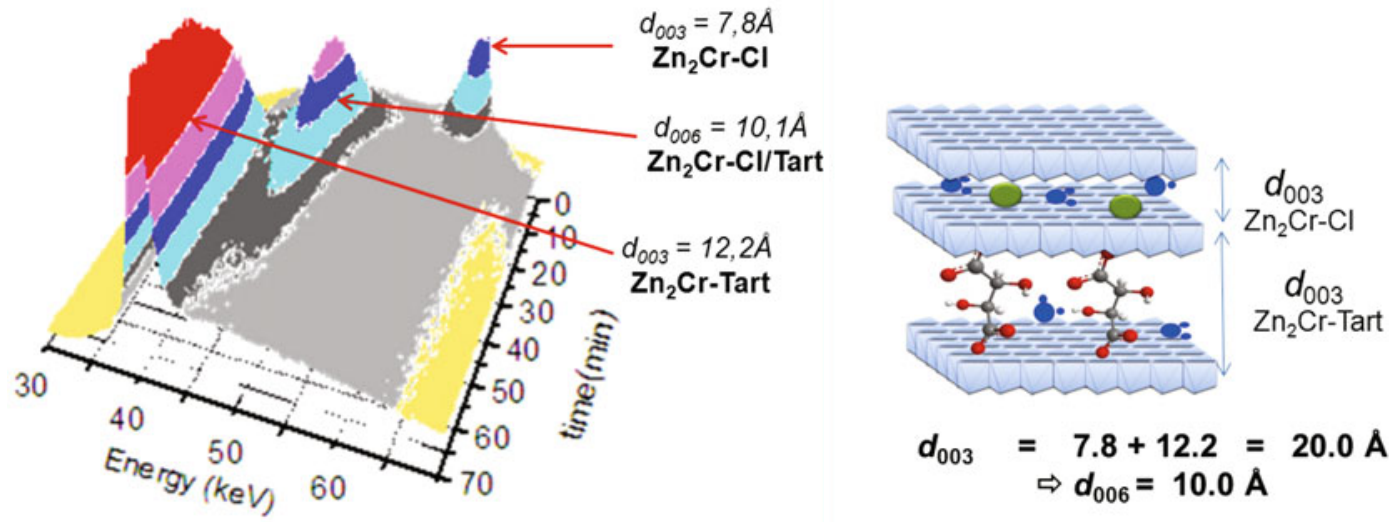

Figure 6. Suivi in situ en solution de la réaction d'échange des anions $\mathrm{Cl}$ - par les anions tartrate dans la phase $\mathrm{Zn}_{2} \mathrm{Cr}(\mathrm{OH})_{6} \mathrm{Cl} .2 \mathrm{H}_{2} \mathrm{O}$. Les mesures ont été réalisées sur la ligne 16,4 du synchrotron SRS à Daresbury (UK) dans les conditions suivantes : suspension de $250 \mathrm{mg} \mathrm{HDL}$ dans $10 \mathrm{~mL}$ eau ; $\mathrm{T}=20^{\circ} \mathrm{C}$; vitesse d'addition de la solution d'anions tartrate $0,2 \mathrm{M}: 0,094 \mathrm{~mL} / \mathrm{min}$; temps d'acquisition : $60 \mathrm{sec} / \mathrm{spectre})$.
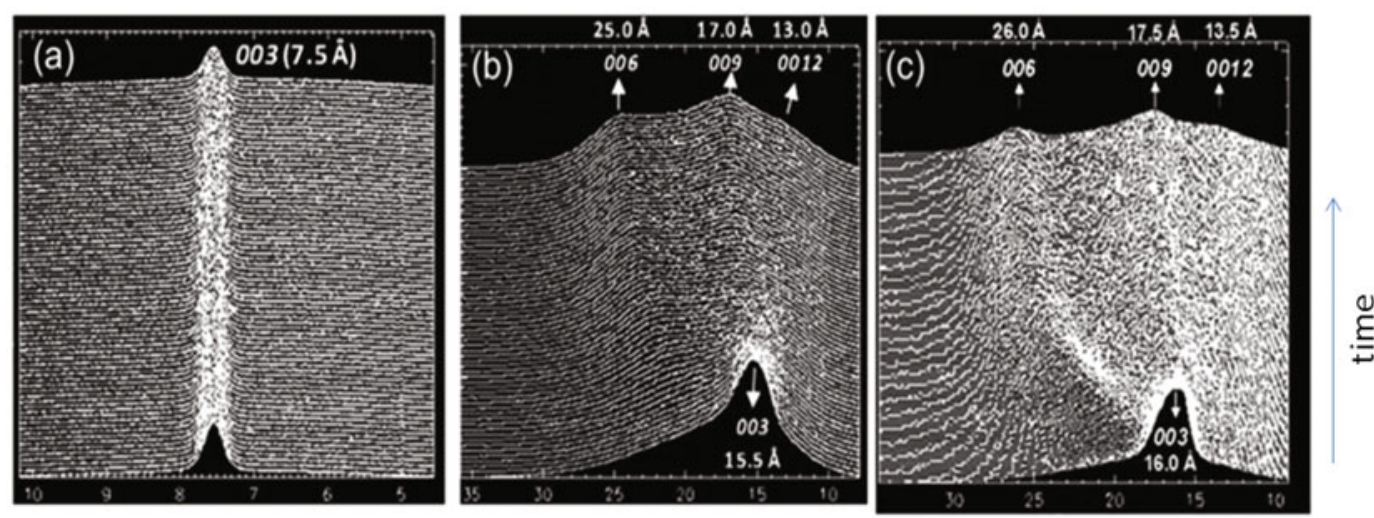

$d(\AA)$

Figure 7. Suivi in situ par EDXRD du mécanisme de dispersion de charges HDL dans une suspension de polyuréthane : (a) $\left[\mathrm{Zn}_{2} \mathrm{Al}(\mathrm{OH})_{6}\right]\left[{ }^{1} / 2 \mathrm{CO}_{3}^{2-}, \mathrm{nH}_{2} \mathrm{O}\right]$, (b) $\left[\mathrm{Zn}_{2} \mathrm{Al}(\mathrm{OH})_{6}\right]\left[4-\mathrm{absa}, \mathrm{nH}_{2} \mathrm{O}\right]$ et (c) $\left[\mathrm{Zn}_{2} \mathrm{Al}(\mathrm{OH})_{6}\right][4-\mathrm{hbsa}$, $\mathrm{nH}_{2} \mathrm{O}$ ]. Les mesures ont été réalisées sur la ligne 16,4 du synchrotron SRS à Daresbury (UK) dans les conditions suivantesc : suspension de $250 \mathrm{mg} \mathrm{HDL}$ dans $10 \mathrm{~mL}$ eau ; $\mathrm{T}=20^{\circ} \mathrm{C}$; vitesse d'addition de la solution de WBPU : $0,094 \mathrm{~mL} / \mathrm{min}$; temps d'acquisition : $60 \mathrm{sec} / \mathrm{spectre})$.

nature différente permet d'envisager l'élaboration d'une nouvelle catégorie de matériaux multicouches et multifonctionnels combinant les propriétés de la structure hôte HDL et celles de deux espèces intercalées.

Le processus de formation des composites HDL-polymère a également été étudié par EDXRD [15]. L'introduction de charges minérales HDL dans les polymères permet d'améliorer les propriétés mécaniques et la tenue thermique de l'ensemble, également de modifier la perméabilité au gaz du polymère. Les mesures présentées Figure 7 concernent la dispersion de trois charges HDL dans du polyuréthane WBPU : $\left[\mathrm{Zn}_{2} \mathrm{Al}(\mathrm{OH})_{6}\right]\left[1 / 2 \mathrm{CO}_{3}^{2-}, \mathrm{nH}_{2} \mathrm{O}\right],\left[\mathrm{Zn}_{2} \mathrm{Al}(\mathrm{OH})_{6}\right]\left[4-\mathrm{absa}, \mathrm{nH}_{2} \mathrm{O}\right]$ (4-absa : 4-aminobenzenesulfonic) et $\left[\mathrm{Zn}_{2} \mathrm{Al}(\mathrm{OH})_{6}\right]\left[4-\mathrm{hbsa}, \mathrm{nH}_{2} \mathrm{O}\right]$ (4-hbsa : 4-hydroxybenzenesulfonic). 


\section{Web of Conferences}

Dans le cas des charges hybrides, la dispersion dans le polyuréthane s'accompagne d'un phénomène d'exfoliation avec un écartement important des feuillets d'environ $35 \AA$ qui a lieu dans les minutes qui suivent l'introduction de la charge HDL dans le polyuréthane. L'examen des films en microscopie électronique à balayage confirme cet état d'exfoliation. Plusieurs études ont montré que le comportement rhéologique des composites HDL-polymères dépend de leur état de dispersion, en particulier de leur niveau d'exfoliation [16].

\section{Remerciements}

Les auteurs remercient vivement L. Aimoz et E. Curtis (Institut Paul Scherrer, Villigen, Suisse), P. Bordet (Institut Néel, Grenoble), D. O'Hare (Chemistry Research Laboratory, University of Oxford, UK) et G. Williams (London Metropolitan University, UK), J.C. Neyt, A. Faour, C. Mousty et Vanessa Prévot (Institut de Chimie de Clermont-Ferrand, Université Blaise Pascal) pour leur contribution essentielle à ces travaux ainsi que E. Elkaim (SOLEIL, Saclay), D. Taylor et T. Bell (SRS Daresbury Laboratory, UK) pour l'aide précieuse lors des expériences synchrotron.

\section{Références}

[1] Layered Double Hydroxides Structure and Bonding vol. 119, X. Duan D.G. Evans (Editors) 2006. Series Editor D.M.P. Mingos, Springer-Verlag, Berlin

[2] L. Aimoz, C. Taviot-Guého, S.V. Churakov, M. Chukalina, R. Dähn, E. Curti, P. Bordet, M. Vespa, J. Phys. Chem. C 116, 5460 (2012)

[3] V. Prevot, N. Caperaa, C. Taviot-Gueho, C. Forano, Cryst. Growth Des. 9, 3646 (2009)

[4] A. Faour, C. Mousty, V. Prevot, B. Devouard, A. De Roy, P. Bordet, E. Elkaim, C. Taviot-Gueho, J. Phys. Chem . C 116, 15646 (2012)

[5] J.C. Neyt Analyse structural de phases HDL : approche combine par simulation moléculaire et diffraction de rayons $X$ (Master Recherche, Université Blaise Pascal, Clermont-Ferrand II, 2010)

[6] J. Rodriguez-Carvajal, Powder Diff. Int. Union Cryst., Newsletter 26, 12 (2001)

[7] P. Thompson, D.E. Cox, J.B. Hasting, Appl. Cryst. 20, 79 (1987)

[8] T. Egami, S. J. L. Billinge, Underneath the Bragg peaks: structural analysis of complex materials (Pergamon Press, Elsevier, Oxford, England, 2003)

[9] X. Qiu, J.W. Thompson, S.J.L. Billinge, J. Appl. Crystallogr. 37, 678 (2004)

[10] T. Proffen, S.J.L. Billinge, J. Appl. Crystallogr. 32, 572 (1999)

[11] J. Pisson, C. Taviot-Gueho, Y. Israeli, F. Leroux, P. Munsch, J.P. Itie, V. Briois, N. MorelDesrosiers, J.P. Besse. J. Phys. Chem. B 107, 9243 (2003)

[12] Y.J. Feng, G.R. Williams, F. Leroux, C. Taviot-Gueho, D. O’Hare, Chem. Mater. 18, 4312 (2006)

[13] G.R. Williams, A.M. Fogg, J. Sloan, C. Taviot-Gueho, D. O’hare, Dalton Trans. 3499 (2007)

[14] C. Taviot-Guého, Y.J. Feng, A. Faour, F. Leroux, Dalton. Trans. 39, 5994 (2010)

[15] A.L. Troutier-Thuilliez, H. Hintze-Bruening, C.Taviot-Gueho, V. Verney, F. Leroux, Soft Matter 7, $4242(2011)$

[16] F. Leroux, J. Nanosci. Nanotechnol. 6, 303 (2006) 